\title{
Untersuchungen \\ über die bakterizide Wirkung der Radiumemanation
}

(sowie Beschreibung eines von Prof. K. Prytz konstruierten Apparates zar Gewinnung der Emanation ans festen Radiumpräparaten).

Von

Privatdozent Dr. med. Hans Jansen

in Kopenhsgen.

(Hierza Taf, IY,)

DaB Radiumemanation bakterizide Eigenschaften besitzt, ist von Danysz ${ }^{1}$, Kalmann ${ }^{2}$, Dorn, Baumann und Valentiner ${ }^{3}$, Rheinbold $^{4}$, Dautwitz ${ }^{6}$, Bouchard und Balthazard ${ }^{6}$ nachgewiesen. Kalmann und Rheinbold benutzten Quellwasser und Quelluft. Die anderen Verfasser bedienten sich der Emanation von Radiumpräparaten.

Die Wirkung ist an Bac. prodigiosus, Bac. pyocyaneus, Bact. fluorescens, Choleravibrionen, Milzbrand-, Typhus-, Mäusetyphus - und Diphtheriebazillen erprobt.

Wachstumsbeschränkung wurde in allen Fällen konstatiert, in einzelnen, wo Emanation aus kräftig wirkenden Radiumpräparaten zur Verfügung

1 Danysz, De l'action pathogène des rayons et des émanations émises par le radium sur différents tissus et différents organismes. Compt. rend. de l'acad. des sciences. 1903. T. CXXXVI. p. 461.

Kalmann, Ein Beitrag zur Kenntnis der Radiumwirkung von Heilquellen. Wiener klin. Wochenschrift. 1905. Nr. 22.

${ }^{3}$ Dorn, Baumann n. Valentiner, Einwirkung der Radiumemanation auf pathogene Bakterien. Diese Zeitschrift. 1905. Bd. LI.

4 Rheinbold, Zar bakteriziden Wirkung radioaktiven Mineralwassers. Berl. klin. Wochenschrift. 1906. Nr. 20.

- Dantwitz, Beitrag zur biologischen Wirkung der radioaktiven Uranpecherzrückstände aus St. Joachimsthal in Böhmen. Zeitschr. f. Heillkunde. 1906. Bd. XXVII.

- Bouchard et Balthazard, Action de l'émanation du radium sur les bactéries chromogènes. Compt. rend. de l'acad. des sciences. T. CXLII. p. 819. 
stand, auch Tötung.: Die meisten der angeführten Arbeiten sind kurze Mitteilungen über vereinzelte Versuche; nur in Dorn, Baumann und Valentiners Arbeit ist die Frage der bakteriziden Wirkung der Radiumemanation eingehender besprochen. Ihre Versuchsanordnung diente mir teilweise als Vorbild. Sie schalteten diejenigen Kulturen, welche dem Einfluß der Emanation ausgesetzt werden sollten, in ein geschlossenes System von Röhren ein. Die Luft in diesem System wurde mittels eines Doppelgebläses in Zirkulation gesetzt und über die auf schrägen Agar ausgestrichene Kultur hinabgeblasen oder durch eine Bouillonkultur getrieben. An einer Stelle im System befand sich das Radiumpräparat in gelöstem Zustande, und von diesem erhielt die Luft ihren Emanationsgehalt. Die Verfasser stellten nicht fest, wie gro $B$ derselbe in den einzelnen Versuchen war, überhaupt beschäftigten sie sich nicht damit, die zur Tötung erforderliche kleinste Dosis zu bestimmen; jedoch ist angeführt, daB 1 Liter der Luft innerhalb 1 Minute $3 \times 10^{7}$ E. S. E. abgeben konnte. (Bei Anwendung von $30^{\mathrm{mg}}$ Radiumbromid, wovon die Emanation 8 Tage nicht entfernt worden war.)

Durch Aufblasen einer derart stark aktiven Luft mehrmals täglich während einiger Tage (2-3-4) wurden Kulturen von Typhus, Mãusetyphus, Cholera und Diphtherie, frisch gesät auf der Oberfläche schrägen Agars sterilisiert; es entstand aber konstant Wachstum im Kondensationswasser. Durchtreiben der radioaktiven Luft durch Typhusbouillonkulturen konnte die Anzahl der Bakterien auf $1 / 3$ hinabbringen (nachgewiesen durch Aussaat in Agar), es gelang jedoch nicht, die Bouillon zu sterilisieren.

Etwas ausführlichere Angaben über den Stärkegrad der angewendeten Emanation finden wir bei Bouchard und Balthazard, z. B. hörte die Pigmentbildung von Bact. fluorescens bei Einwirkung der sich in 1 Stunde aus $0.0006^{\mathrm{mg}}$ Radiumbromid entfaltenden Emanation auf. Das Wachstum stockte erst bei einer. Emanationsmenge (in 1 Stunde entwickelt) von $0.15^{\mathrm{mg}}$ Radiumbromid. Kulturen von Bac. pyocyaneus wurden durch Einwirkung der in 4 Tagen aus $25^{\text {mg }}$ Radiumbromid entwickelten Emanation getötet. Leider geben die Verfasser keine nähere Beschreibung der Versuchsanordnung. Dies in Verbindung mit der indirekten Art der Angabe der Emanationsmenge erschwert einen Vergleich dieser Versuche mit den meinigen.

Außer den angeführten Arbeiten mit positivem Resultat gibt es aus dem Jahre 1908 eine einzelne von Suess ${ }^{1}$ mit gänzlich negativem Befund.

' Suess, Über den EinfluB der Radiumemanation auf Tuberkelbazillen and auf experimentelle Tuberkulose. Zeitschrift f. Tuberkulose. 1908. Bd. XII. S. 480. 
Indessen verfügt der Verfasser über gar keine Messungen der angewendeten Emanation; in Wirklichkeit weiB er nicht, ob seine Kulturen überhaupt ron Emanation beeinfluBt sind. Allenfalls kann diese nicht sonderlich stark gewesen sein, da das benutzte Präparat $\left(0.1663^{\mathrm{mg}}\right.$ Radiumchlorid) nur eine Aktivität von 40000 Uraneinheiten auswies. Die wesentlichsten seiner Versuche gelten Tuberkelbazillenkulturen, welche er in 2 Tagen mit der emanationshaltigen Luft behandelte und darauf teils übersäte, teils auf Meerschweinchen einimpfte. Sie erwiesen sich als gänzlich ungeschwächt. Die Versuche wurden noch auf verschiedene Art variiert, im großen und ganzen aber mit demselben negativen Resultat.

Völlig negative Resultate erbielt auch Loewenthal-Braunschweig ${ }^{1}$, der Aufschwemmungen ron Bac. prodigiosus und ron Bact. coli, sowie von Staphylokokken durch 1 bis 3 Stunden im Brutschrank mit Emanation vorbehandelte (Radiogenwasser mit einem Gehalt von 2000 Volt) und dann zur Aussaat brachte. Loewenthal erklärt die positiven Resultate anderer Autoren einmal mit den viel höheren Emanationsmengen, andererseits mit der dadurch bedingten Ozonentwicklung.

Abgesehen von den hier angeführten, vereinzelten Angaben sind, soviel ich weiß, bisher keine Untersuchungen veröffentlicht über das Verhältnis zwischen der Stärke der angewendeten Emanation und der erzielten Wirkung (auf Bakterien); ebenfalls kein Versuch mit systematisch rorgenommenen Messungen der angewendeten Emanation nach den nun gebräuchlichen Methoden. Da unsere eingehendere Kenntnis dieses Verhältnisses sowohl aus rein theoretischen Gründen wie im Hinblick auf eine eventuelle Anwendung in der Therapie der Radiumemanation von Wichtigkeit ist, habe ich diese Frage in Angriff genommen, was um so leichter fiel, da mir seitens des Hrn. Professors des hiesigen Polytechnikums K. Prytz ein von ihm konstruierter Apparat überlassen wurde, der sich ausgezeichnet zu Untersuchungen diẻser Art eignete.

Ich habe versucht, folgende Fragen zu lōsen:

1. Wie radiumemanationshaltig $m u B$ die Luft sein, um absolut tötend auf eine frisch gesäte Oberflächenkultur wirken zu kōnnen?

2. Von wie langer Dauer muB die Einwirkung sein?

3. Welches ist die geringste Dosis Emanation, die eine eben erkenubare Wirkung auf Bakterien hervorruft?

${ }^{1}$ Isoewenthal-Braunschweig, Über die Wirkung der Radiumemanation auf den Menschen. 4. Mitteilung. Berliner klin. Wochenschrift. 1910. Nr. 7. 
Es ist nur versucht worden, diese Fragen hinsichtlich einer einzigen Bakterienart, nämlich Bac. prodigiosus, zu lösen.

Die benutzte Radiumemanation stammt von $16^{\text {mg }}$ Radiumbaryumbromid mit einer Aktivität ron 500000 Uraneinheiten. Dieses Präparat liegt in einer aus einer Glas- und einer Ebonitplatte bestehenden Kapsel, welche durch eine Metallfassung zusammengeschroben gehalten wird. Professor Prytz konstruierte den angeführten Apparat, um die Emanation

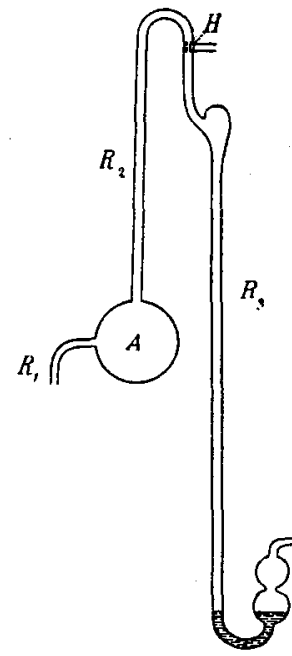

Fig. 1.

Schematische Darstellung des Prytzschen Apparates. gewinnen zu können, ohne ein Öffnen der Kapsel zu benötigen. Der Apparat ist folgendermaßen eingerichtet (siehe Fig. 1).

$A$ ist ein Metallbehälter mit dicken Wänden; die Vorderwand ist abschraubbar. Die Kapsel mit dem Radiumpräparat wird in diesen Behälter gelegt, und die Vorderwand luftdicht zugeschroben. Von dem Behälter gehen 2 Glasrohre luftdicht aus; das eine $\boldsymbol{R}_{1}$, steht in direkter Verbindung mit einer Prytz-Schlauchpumpe ${ }^{1}$ (die saugende Mündung), das zweite Rohr $R_{2}$ geht erst aufwärts und darauf in ein $63^{\mathrm{cm}}$ hohes, oben etwas erweitertes Rohr $R_{3}$ über; unten biegt dieses $\operatorname{Rohr} R_{3}$ in einen kleineren Behälter ein, und dieser wird durch einen Schlauch mit der anderen Mündung der Schlauchpumpe in Verbindung gesetzt. Derart bildet das ganze einen geschlossenen Ring, und durch Drehen der Pumpe wird nur die im Behälter und in den Rohren eingeschlossene Luft in Zirkulation gesetzt.

Im Rohr $R_{3}$ befindet sich eine Quecksilbersäule. In der Ruhestellung wird sie, wie auf der Zeichnung vermerkt, teils in dem unteren Bogen liegen, teils in dem Behälter, in welchem dieser Bogen mündet. Beim Pumpen wird die Luft aus dem Behälter $A$ herausgesogen, das Quecksilber wird daher im Rohr $R_{3}$ steigen, bis es an die Erweiterung gelangt. Indem die Säule hier verkürzt wird, drängt die Luft unter derselben durch das Quecksilber hinauf, der negative Druck in $A$ und über dem Quecksilber hört auf, und das Quecksilber fällt wiederum zu Boden im Rohr $R_{3}$. Bei fortgesetztem Pumpen wiederholt sich dieser Vorgang unaufhörlich. Auf diese Weise stehen im Behälter $A$ zahlreiche, aufeinanderfolgende Unterdrucke. Ihre GröBe hängt von der Höhe der Quecksilbersäule $a b$; in der Regel betrug diese $22 \mathrm{~cm}$.

1 Beschrieben in Zeitschrift für Instmumentenkunde. 1905. S: 193. 
Es soll noch hinzugefügt werden, daB sich am Ubergang $z$ wischen $R_{2}$ und $R_{3}$ ein Hahn $H_{1}$ mit dreiteiliger Bohrung und einern Seitenrohr befindet. Es lassen sich hier Proben der Apparatluft nehmen, und überdies kann man hierdurch das obere Ende von $R_{3}$ mit der saugenden Mündung der Schlauchpumpe in direkte Verbindung setzen und die Luft außen um den Behälter $A$ zirkulieren lassen.

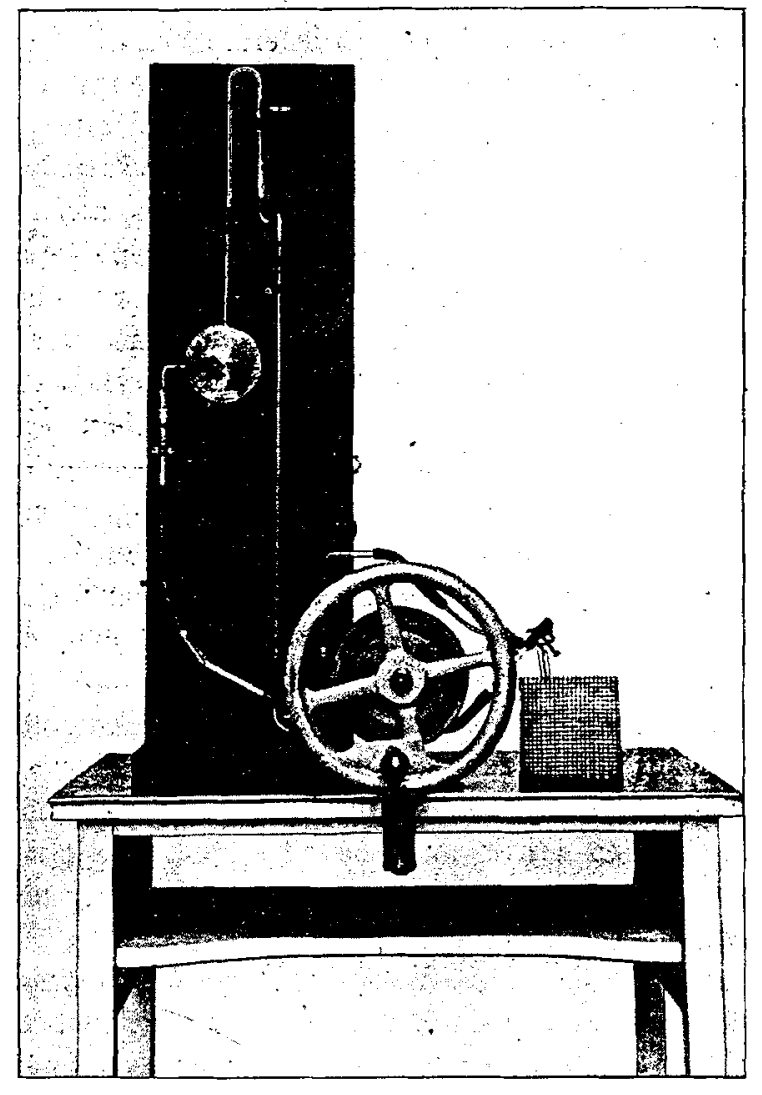

Fig. 2.

Photographie der Gesamtanfstellung.

Liegt die Radiumkapsel an ihrem Platz in $A$ und es wird gepompt, so geschieht folgendes: Bei jedem Unterdruck wird etwas von der inwendig das Radiumpräparat umgebenden emanationsgesättigten Luft durch die Schraubengewinde der Kapsel hinausgesogen. Da es beständig dieselbe Luft ist, welche zirkuliert, wird diese allmählich mit dem Pumpen emanationsreicher werden. 
Wie emanationsreich die. Apparatluft zu werden vermag, hängt in erster Instanz von dem Zustand des Radiumpräparates wãhrend des betreffenden Zeitpunktes $a b$. Bekanntlich spaltet Radium unaufhaltsam Emanation ab. Ein Teil derselben verläBt das Radiumpräparat, aber ein Teil häuft sich darin auf. Diese Anhäufung schreitet fort, bis ein gewisser Gleichgewichtszustand erreicht ist. Das Präparat ist dann maximal mit Emanation beladen. Wird diese Ladung verbraucht, so vergeht ungefähr 1 Monat, bevor die maximale Ladung wiederum erreicht ist. Von dieser angehäuften Emanation entnimmt man beim Pumpen mit dem Prytzschen Apparat und wird mithin die emanationsreichste Luft erhalten Können, wenn das Präparat maximal beladen ist, und gradweise weniger, je mehr entnommen und je kürzere Zeit seit der letzten Abnahme verstrichen ist.

Wie lange in dem einzelnen Fall zur vollen Gewinnung der ganzen zur Verfügung stehenden Emanationsmenge gepumpt werden soll, ist einigermaßen von der Funktion der Pumpe, namentlich der Häufigkeit des Unterdruckes in der Minute und seiner Größe abhängig; in der Regel geht es aber recht schnell. Versuche ergaben, daB das gröBte Quantum der Emanation schon nach 4 bis 5 Minuten Pumpen entwichen war; wurde über 15 Minuten hinaus gepumpt, so nahm der Emanationsgehalt der Apparatluft nicht mehr wesentlich zu. Um ganz sicher zu sein, pumpte ich doch gewöhnlich 30 Minuten, wenn ich volle Wirkung wünsehte.

Diese Beschreibung läBt uns verstehen, daB die zwischen zwei Entnahmen verstreichende Zeit für das Emanationsquantum von größerer Bedeutung ist, als die Dauer des Pumpens. Durch halbstūndiges Pumpen nach z. B. 24 Stunden erhält man dasselbe Emanationsquantum in der Apparatluft, wie bei ununterbrochenem oder wiederholtem Pumpen während dieser 24 Stunden.

Bei der von mir benutzten Versuchsanordnung, wo eine Bakterienoberflächenkultur in den genannten Kreislauf eingeschaltet wurde, zeigte es sich nun, daß die Wirkung bei übrigens derselben Emanationsmenge bei mehrmaligem Pumpen während der Versuchsdauer gröBer als bei einmaligem Pumpen war. Zuzuschreiben ist diese Erscheinung wahrscheinlich dem direkten Aufblasen und dem Luftwechsel, wodurch die ganze, in der Apparatluft vorhandene Emanation die Kultur passierte, während beim Stehen nach dem Pumpen nur die über der Kultur stehende Emanation auf die Kultur wirkte.

Zum Drehen der Pumpe wurde ein elektrischer Motor benutzt. Die Geschwindigkeit wurde teils durch Rheostat, teils durch Schnürzugübertragung reguliert. Gewöhnlich machte das Rad der Schlauchpumpe etwa 
100 Umdrehungen in der Minute, wobei sich das Quecksilber etwa $12 \mathrm{mal} \mathrm{hob.}$

Als Objekt für die Einwirkung der Emanation habe ich Bac. prodigiosus benutzt. Ich wählte diese Bakterie, teils weil sie so leicht zu erkennen ist, was die Kontrolle der Reinheit der Kultur leicht macht, teils weil ich aus den so verwandten Versuchen über die bakteriziden Eigenschaften des Lichtes gewohnt gewesen bin mit dieser Bakterie zu arbeiten.

Die Emanation als solche entsendet nur $\alpha$-Strahlen, welche bekanntlich sehr wenig penetrant sind. Es war demnach von vornherein zu erwarten, daB die bakteriziden Wirkungen der Emanation nicht recht tief gingen, was auch die Versuche von Dorn, Baumann und Valentiner bekräftigen; es zeigte sich bei diesen, daß nur die 2 oberen Millimeter einer Aussaat in Gelatine sterilisiert wurden, unter Einwirkung stark emanationshaltiger Luft über der Kultur. Ich benutzte deshalb Oberflächenkulturen auf folgende Weise.

In sterile Präparatgläser von etwa $10^{\mathrm{cm}}$ Höhe, etwa $3 \mathrm{~cm}$ im Diameter, tat ich etwa $6 \mathrm{~cm}$ steriles Agar, welches ich in schräger Stellung gerinnen lie日, so daß die Fläche mit dem Boden einen Winkel von $45^{\circ}$ bildete. Diese Fläche besäte ich mit einer 20 bis 24 Stunden alten Bouillonkultur des Bac. prodigiosus. Über die Fläche wurde eine bestimmte Anzahl Tropfen der veräünnten Kultur gegossen. Die sich am FuBe der Fläehe sammelnde überschüssige Flüssigkeit saugte ich unter Anwendung einer sterilen Pasteurpipette fort. (Dies geschah aber nicht in den ersten Persuchen.)

Das Versuchsglas wurde darauf mit einem doppelt durchbohrten Gummipfropfen geschlossen. Durch das eine Loch führte ein Glasrohr tief hinab ins Glas, so daß die Mündung nur 1 bis $2 \mathrm{~cm}$ über der Mitte der Agarfäche zụ liegen kam. Durch die andere Bohrung ging ein kurzes Rohr nur gerade hindurch. Die freien Enden der Rohre waren mit Gummischläuchen und Klemmhähnen versehen.

Das so präparierte Versuchsglas schaltete ich zwischen dem von der Schlauchpumpe führenden Ende und dem Emanator ein; der Luftstrom wurde durch das lange Rohr geleitet und also beim Pumpen direkt hinab über die Kultur geblasen. Die Luft passierte ein Wattefilter. Nach beendigtem Pumpen wurden die Klemmhähne zugeschraubt, und das Versuchsglas konnte herausgenommen und in den Thermostat gesetzt werden $\left(24^{0}\right)$. In einer bestimmten Reihe Versuche wurde die Emanation nach einer gewissen Zeit durch Durchsaugen frischer Luft entfernt, und den Gummipfropfen ersetzte ein Wattepfropfen. In mehreren Versuchen 
wurden gleichzeitig zwei Versuchsgläser hintereinander eingeschaltet. Nach beendigter Behandlung wurde die Emanation aus dem einen, nicht aus dem anderen entfernt. In den Fällen wo im Laufe von 24 bis 48 Stunden wiederholt gepumpt wurde, blieben die Versuchsgläser im Apparat stehen, also bei Stubentemperatur.

$\mathrm{Zu}$ jedem Versuch wurde aus derselben Kulturverdünnung ein Kontrollglas auf vollkommen gleiche Weise zubereitet. Dieses wurde auch mit dichtschlieBendem Gummipfropfen versehen.

Die Messung des Emanationsgehaltes oder der Radioaktivität der Luft geschah mittels eines Sievekingschen Fontaktoskopes. ${ }^{1}$ Da dieses Fontaktoskop sehr empfindlich ist, weil es zur Messung der recht kleinen Mengen Emanation dient, welche in Quellen vorkommen, durfte ich nur kleine und eventuell verdünnte Proben der Iuft des Apparates einfübrea.

Diese Luftproben wurden durch Saugen mit einer Lūerschen 10 com Glasspritze durch das Seitenrohr $S$ und den Hahn $H$ entnommen. In der Regel wurde 1 cem der Apparatiuft in der Spritze mit 9 com gewöhnlicher Luft verdünnt und davon nur die Hälfte in das Fontaltoskop eingeführt, also nur $1 / 2{ }^{\mathrm{cm}}$ Apparatluft. Bei weniger aktiver Luft wurde 1 oder sogar 2 bis $4^{\mathrm{ccm}}$ der unverdünnten Apparatluft eingeblasen.

Die Luftprobe wurde durch das Mundstück unten am Fontaktoskop eingeblasen, das mit aufgesetztem und geladenem Elektroskop dastand. Die Aluminiumblätter begannen augenblicklich zusammenzufallen, und in der Regel wurde das Elektroskop in weniger als 1 Minute entladen. An der eingeteilten Skala des Elektroskops lieB sich z. B. der Zusammenfall in $1 / 2$ Minute ablesen. Nach einer Tabelle wurden die erhaltenen Werte in Volt umgesetzt und diese durch Multiplizieren zur Gültigkeit für 1 cem und 1 Stunde umgerechnet. Die derart gefundenen Zahlgrößen lagen in der Regel hoch in den Tausenden.

Gegenüber diesen schnellen Entladungen spielte der Normalverlust des Elektroskops gar keine Rolle, weshalb ich ihn gewöhnlich bei der Ausrechnung unberücksichtigt ließ - er soll subtrahiert werden. Ieh überzeugte mich nur immer, ob die Blätter vor dem Versuche in Ruhe waren. Durch schnelles Arbeiten und sofortige Entfernung der zugeführten Emanation durch Auffüllen von Wasser, gelang es mir trotz sehr zahlreicher Messungen, den Normalverlust des Apparates auf einem Minimum zu halten (um 50 Volt per Stunde herum).

1 Sieveking, Über einen neuen Apparat zur Bestimmung der Radioaktivität der Thermalquellen. Phys. Zeitschrift. 1905. Bd. VI. S. 700. 
Die gefundenen Werte von mehr als 10000 Voltverlust rundete ich auf ganze Tausend $a b$, da die Differenzen von einigen Hundert absolut keine Rolle spielten, und die Messungen selbst - der hohen Zahlen wegen - die Möglichlieit für Fehler derselben GröBe nicht ausschlossen. Diese abgerundeten Angaben von Voltverlust per $1^{\mathrm{com}}$ und Stunde, habe ich schlieBlich in Macheeinheiten umgerechnet, d. h.: elektrostatische Einheiten multipliziert mit 1000. Dies geschah nach der Formel: $\frac{V}{300} \cdot \frac{13 \cdot 8}{3600}=$ E.S.E., wo 13.8 eine für das betreffende Fontaktoskop geltende konstante GröBe ist, $300=1$ E.S. E., $3600=$ Sekunden 1 Stunde. Durch dieses Umrechnen erhalten die Zahlen konstanten Wert, unabhängig vom MeBinstrument.

Die Untersuchungen über die 3 in der Einleitung aufgeworfenen Fragen sind durcheinander im Laufe der letzten $1 \frac{1}{2}$ Jahre ausgeführt. Hier habe ich jedoch die Versuche in drei Gruppen, den drei Fragen entsprechend, geordnet und sie tabellarisch aufgestellt. In einigen Fällen sind. Versuche der Gruppen I und II gleichzeitig gemacht, indem ich gleichzeitig zwei Versuchsglāser in die Pumpe einschaltete. Die Emanation wurde dann nach einiger Zeit von dem einen Glas entfernt, nicht von dem anderen. So gehören folgende Versuche zusammen: Gruppe I, Nr. 5 und II, Nr. 6, Gruppe I, Nr 8 und II, Nr. 7.

Gruppe I (siehe S. 144). Vor allem besagen diese Versuche, daB Bac. prodigiosus von Radiumemanation getötet werden kann. Jedoch ist die sterilisierende Fähigkeit der Emanation - in den angewendeten Stärken - nicht sonderlich groB. Die Emanation vermochte nur mit der ganz dünnen Schicht frisch gesäter Kulturen fertig zu werden. Es gelang nicht, das Kondensationswasser zu sterilisieren (analog den Versuchen von Dorn, Baumann und Valentiner). Die gerade erforderliche Dosis zur Sterilisation der Agarfäche lag bei 345 Macheeinheiten (27000 Voltfall pro Stunde) pro $1^{\mathrm{cm}}$ Luft; sie genügte aber nur, wenn das Pumpen (Aufblasen) einigemale wiederholt wurde, selbst wenn die Dosis schlieblich etwas sank, während bei einer einzelnen Pumpperiode die Randpartien nicht vollständig sterilisiert wurden. Die sicher tödliche Dosis für eine Oberflächenkultur von Bac. prodigiosus kann man nach diesen Versuchen auf ca. 400 Macheeinheiten (ca. 32000 Voltfall pro Stunde) pro $1^{\mathrm{ccm}}$ der Luft setzen, welcher die Kultar exponiert ist.

[ $\mathrm{Zu}$ jedem dieser Versuche wurde ein Kontrollglas ganz gleich dem Versuchsglas zubereitet. Verschließung mit Gummipfropfen. In allen Fällen entstand gleichmäBiges Wachstum mit blaß graulichrotem Pigment über der ganzen Fläche. (Die Unvollständigkeit der Pigmentation ist dem hermetischen AbschluB zuzuschreiben.)] 


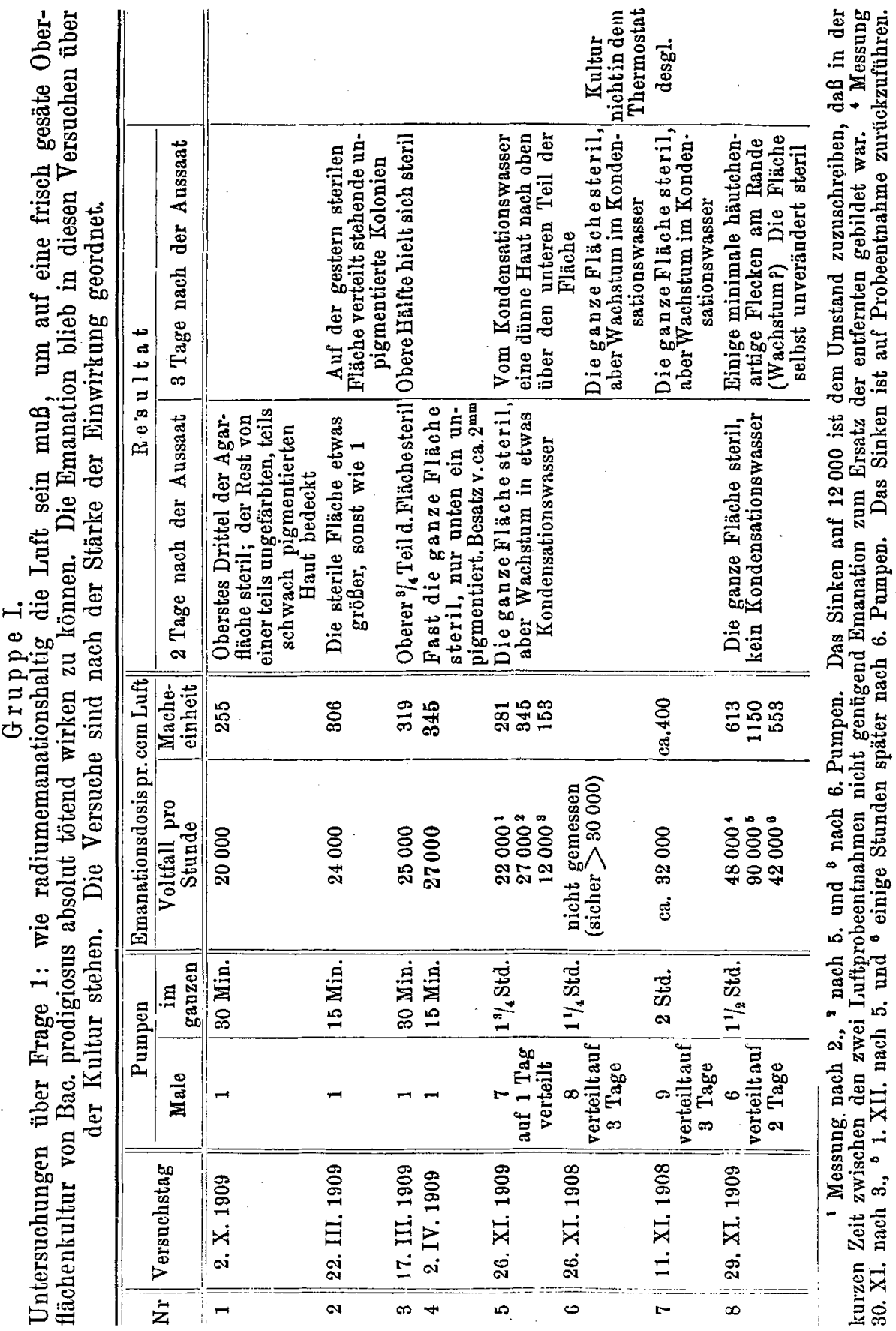




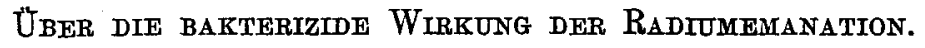

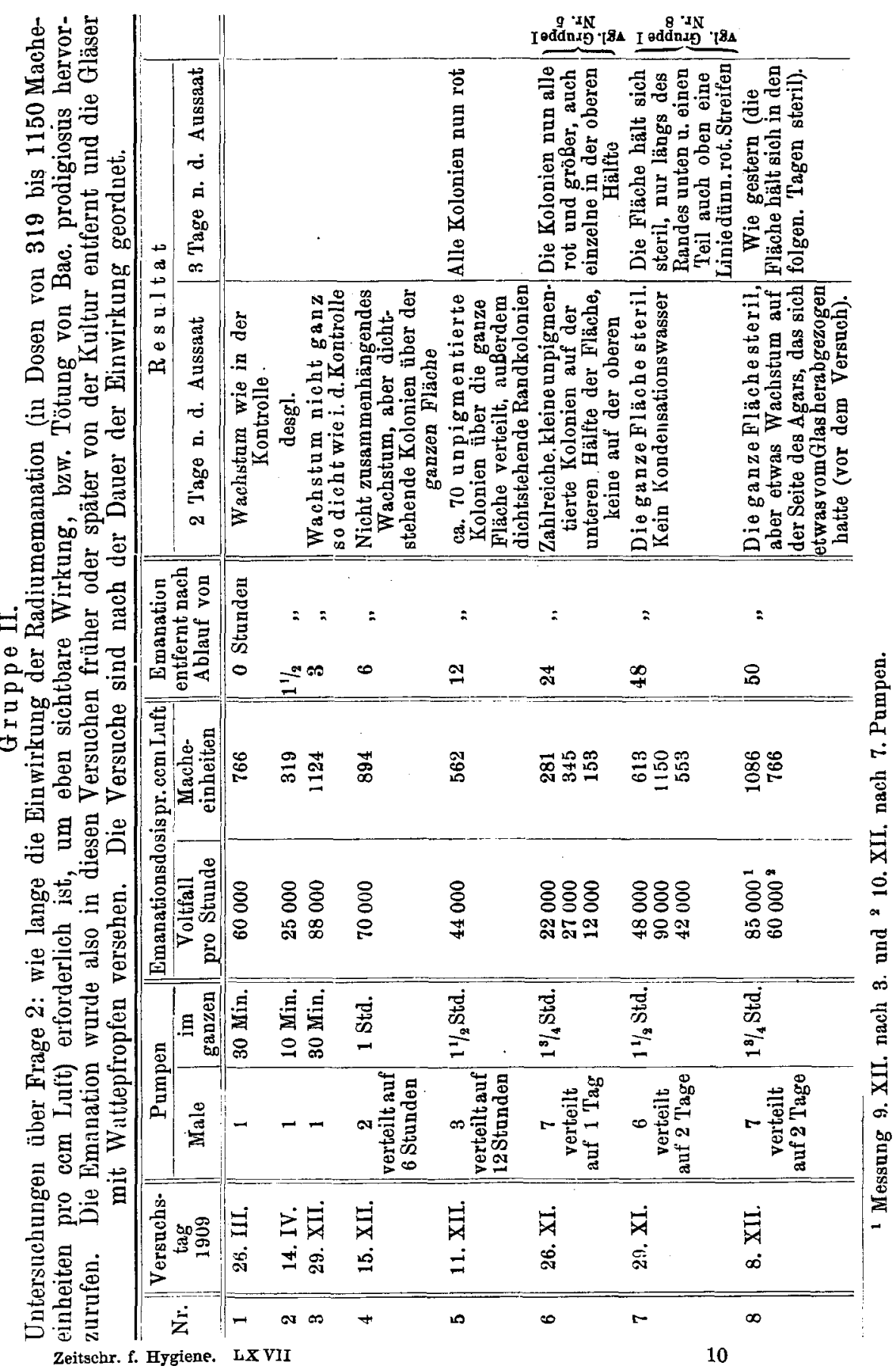


Gruppe II (siehe S. 145): Das Resultat dieser Versuche ist, daB die Radiumemanation - in den angewendeten Dosen - keine momentane Wirkung besitzt. Im Gegenteil scheint ein relativ lang dauerndes Einwirken notwendig zu sein. Trotz 3stündiger Behandlung mit einer sehr stark radioaktiven Luft entstand nur eine eben sichtbare Wachstumsstockung. Wiederholtes Pumpen in 12 und 24 Stunden mit Dosen, welche absolute Tötung ergaben, wenn die Emanation über der Kultur stehen blieb, hatte hier, wo die Emanation entfurnt wurde, nur eine teilweise Sterilisation zur Folge, indem einzelne Kolonien verteilt über die Agarfläche hervorkeimten. Erst nach 48 stündiger Einwirkung einer Luft, deren Radioaktivität um $766^{\circ}$ Macheeinheiten pro Kubikzentimeter schwankte $(60000$ Voltfall in der Stunde), wurde absolute Sterilisation der ganzen Fläche erzielt.

Nach diesen Versuchen ist die Bedeutung einer lange dauernden Einwirkung zweifellos, und, falls die Kulturen während der Dauer der Behandlung mit Emanation nicht in geschlossenen Räumen gehalten würden, müßte die Einwirkung sich wahrscheinlich über eine noch längere Zeit erstrecken. Der LuftabschluB der Kulturen ist ja, woran man immer erinnern muB, ein leicht schädliches Moment, wie es auch an der schlechten Pigmentation in den Kontrollkulturen zu ersehen ist. Eine Nichteinschließung der Kulturen würde ungemeine Mengen Emanation erfordern.

[Zu jedem dieser Versuche wurde ein Kontrollglas, gan\% gleich dem Versuchsglas, zubereitet. Sie waren mit Gummipfropfen geschlossen in derselben Zeit, wie die Emanation in den Versuchsgläsern stand. Es entstand in allen ungefähr gleichmäBiges Wachstum mit kräftig rotem Pigment über der ganzen Fläche.]

Gruppe III (siehe S. 147). Diese Versuche zeigen, daB die Aktivität stillstehender Luft zur Herrorrufung einer eben sichtbaren Wirkung wenigstens 127.5 Macheeinheiten im Kubikzentimeter Luft (10000 Voltfall in der Stunde) repräsentieren muB.

[Die Versuche 7 und 8, eine Folge des Versagens der Pumpe, scheinen dafür zu sprechen, daB sich diese Dosis etwas reduzieren läBt, wenn die Luft wiederholt und längere Zeit über die Kultur geblasen wird.]

[ $\mathrm{Zu}$ jedem dieser Versuche wurde ein Kontrollglas, ganz gleich dem Versuchsglas zubereitet. AbschluB mit Gummipfropfen. In allen Fällen ungefähr gleichmäBiges Wachstum mit blaß graulichrotem Pigment.]

Ferner geht teils aus diesen Versuchen (Nr. 5, 6, 7 und 8), teils aus den Versuchen 1 bis 5 in Gruppe $I$ und 5 bis 6 in Gruppe II hervor, da $B$ die Fähigkeit des Bac. prodigiosus, Pigmentzu bilden, von der Radiumemanation beeinflubt wird. Wohl verringerte die EinschlieBung mit ihrer daraus folgenden weniger guten Aufnahme von 
Uber die bakterizide Wirkung DeR Radiumemanation. 147

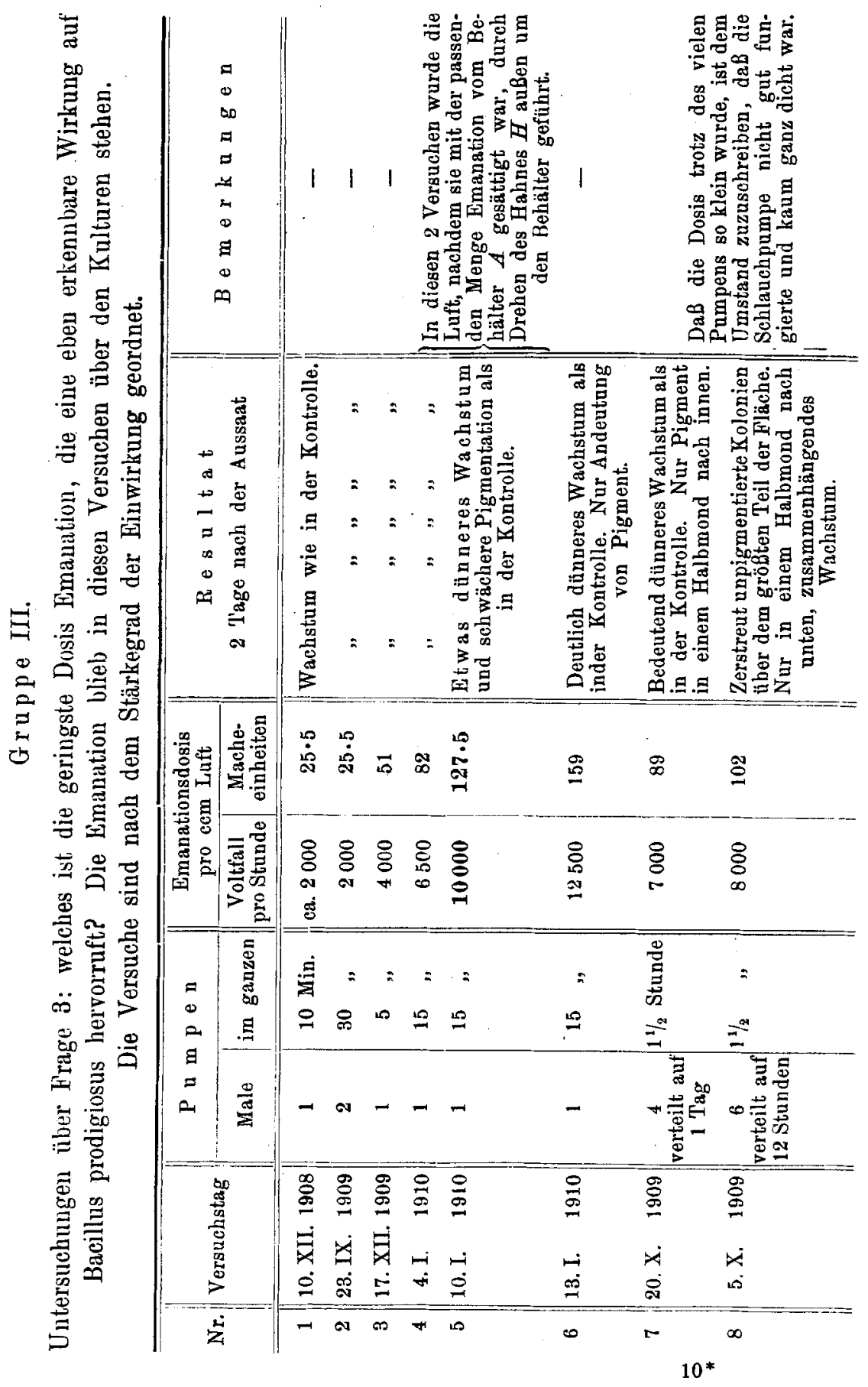


Sauerstoff die Pigmentbildung, was, wie erwähnt, in sämtlichen Kontrollgläsern zur Gruppe I und III zu sehen war; trat aber die Emanation hinzu, so konnte die Pigmentbildung vollständig aufhören, und man erbielt ganz farblose Kolunien oder Wachstuminseln. Hörte die Einschließung auf, wie in Gruppe II, so hörte auch die Hemmung der Pigmentation auf; die Kolonien wurden allmählich vollkommen rot. Bouchard und Balthazard schreiben (in der früher zitierten Arbeit) über Prodigiosus, daB die trotz Einwirkung der Emanation auswachsenden Bakterien ungeschwächte Pigmentation besitzen. Bezüglich dieses Punktes kann ich diesen Verfassern nicht beistimmen.

Ubrigens ist das gefundene Verhältnis ja keineswegs merkwürdig; denn, wie es scheint, wird jedes auf das Wachstumsverhältnis von Bac. prodigiosus hemmend einwirkende Agens auch auf dessen Pigmentation influieren; man weiß es von Wärme, Licht, Sauerstoffmangel, Giftstoffen u. dgl.

Die Versuchsresultate werden vorzüglich durch die ieigefügten halbschematischen Zeichnungen (vgl. Taf. IV, Fig. 1 bis 8) einer Anzahl Kulturen illustriert:

Fig. 1 und 4 zeigen zwei Kontrollkulturen, bzw. zur Gruppe I und Gruppe III, wo die Gläser verschlossen und die Pigmentierung deshalb weniger vollkommen, und zur Gruppe II gehörend, wo die Pigmentierung unbeschädigt war.

Fig. 2 zeigt Versuch 1, Gruppe I; man sieht das dünne hautähnliche Wachstum, das immer bei den Fällen mit nicht genügend intensiver Einwirkung entstand.

Fig. 3 zeigt die vollständige Sterilisation der Agarfläche, die in Versuch 8, Gruppe I durch 2 tägige Einwirkung von Luft mit ca. 60000 Emanationseinheiten pro Kubikzentimeter erreicht wurde.

Fig. 5, 6 und 7 gehören zu Gruppe II unà zeigen die verschiedenen Stadien nach der Dauer der Einwirhung. Fig. 5 zeigt (Versuch Nr. 4), wie wenig eigentlich trotz 6 stündiger Einwirkung einer stark radioaktiven Luft erreicht ist. In Fig. 6 (Versuch Nr. 6) ist man zwar um etliches weiter gekommen, aber die Fläche ist doch noch lange nicht steril, trotz 24 stündiger Einwirkung. Erst nach 48 stündiger Einwirkung einer Luft mit ca. 60000 Emanationseinheiten pro Kubikzentimeter ist es gelungen, eine vollkommen sterile Fläche zu bekommen, wie es Fig. 7 (Versuch Nr. 7) zeigt, längs den Kanten aber sieht man noch Spuren ron Wachstum. 
SchlieBlich repräsentiert Fig. 8 ein Bild des wesentlichsten Versuches in Gruppe III, nämlich Nr. 5; wir sehen leichte Pigmentschwächung und vielleicht etwas dünneres Wachstum als Folge eines Gehalts von 10000 Emanationseinheiten pro Kubikzentimeter der über der Kultur stehenden Luft.

Um zu untersuchen, ob nicht die Versuchsanordnung (abgesehen von der besprochenen EinschlieBung) eine schädliche Einwirkung auf die Kultur hatte, machte ich folgende Kontrollversuche.

1. Durchsaugen gewöhnlicher Luft gleichzeitig mit dem Pumpen. Kulturen, wie gewöhnlich zubereitet, wurden mit einer Wassersaugpumpe in Verbindung gesetzt, so daB die Luft in das lange Rohr hineinging und direkt hinab auf die Kultur. Das Wachstum war ebenso üppig wie in nicht behandelten Gläsern, und doch war die Möglichkeit der Eintrocknung hier gröBer als im Versuch, wo mit abgeschlossener und überdies absichtlich feucht gehaltener Luftmenge gearbeitet wurde.

2. Einwirkung von Quecksilberdämpfen ebenso wie im Versuch.

A priori ist nichts darūber bekannt, inwiefern Quecksilberdämpfe bei Stubentemperatur schädlich auf Bakterien einwirken. Om es doch zu prüfen und überhaupt einige Kulturen einer ganz entsprechenden Behandlung nur mit AusschlieBung der Radiumemanation auszusetzen, stellte ich mir einen Apparat zusammen gleich dem Prytzschen und mit einer anderen Schlauchpumpe, die mir gütigst von Finsens med. Lichtinstitut überlassen wurde. Die Kultur, welche in diesem Apparat im ganzen 2 Stunden behandelt wurde, auf ein paar Tage verteilt, wuchs vollständig unbeeinfluBt aus. Dasselbe Resultat ergab ein Versuch, den ich einmal mit meinem gewöhnlichen Apparat vornehmen konnte, da er unbenutzt und ohne Radiumpräparat 4 Monate lang gestanden batte. Nach einer gründlichen Auslüftung der Rohre zeigte die Apparatluft alsdann nur eine ganz rerschwindende Aktivität.

Die in der Tabelle ausgeführten Resultate lassen sich deshalb nur dem Vorhandensein der Radiumemanation zuschreiben.

Die Wirkung beruht nicht auf einer Veränderung des Nährsubstrates. Dies bewiesen schon die zahlreichen Fälle, wo ein Teil der Agarfläche nach Beendigung des Versuches rom Kondensationswasser infiziert wurde. AuBerdem ersah man es aus folgendem Versuch:

Ein Glas mit Agar, ebenso wie gewöhnlich rubereitet, aber ohne Bakterien, wurde gleichzeitig mit dem Versuchsglas des Versuches Nr. 8 Gruppe II in die Pumpe eingeschaltet; 24 Stunden nach der Entfernung der Emanation wurde die Agarfläche besät, und es entstand gänzlich ungeschwächtes Wachstum. 
150 HANS J ANSEN: Über D. BakTeRIZIDE WIRKUNG D. RADiUMEMANation.

Wabrscheinlich ist die Bakterizidität eine direkte Wirkung und in erster Linie den von der Radiumemanation selbst entsandten $\alpha$-Strahlen zuzuschreiben; hinzu tritt aber sicher auch eine Finwirkung der weniger aktiven festen Stoffe, Radium A, B, C, D, E und F, die sich wie ein Beschlag auf alle Wände des Behälters setzen und demnach auch auf die Kultur. Vielleicht erhält die Kultur etwas mehr dieses Beschlages, wenn die Luft geradezu über sie hinabgeblasen wird; man versteht dann leicht die Bedeutung des häufig wiederholten Pumpens. Eine indirekte Wirkung z. B. durch Ozonbildung aus dem Sauerstoff der Luft ist jedoch nicht auszuschlieBen; hierüber geben diese Versuche an und für sich keine Aufklärung.

\title{
Erklärung der Abbildungen.
}

(Taf. IV.)

\begin{abstract}
Halbschematische Darstellung der Wirkungen von Radiumemanation auf Bacillus prodigiosus.
\end{abstract}

Fig. 1 n. 4 dienen zur Kontrolle; in betreff der übrigen Figuren siehe S. 148. 
Zeitschrift für Hygiene. Bd. LXVII.
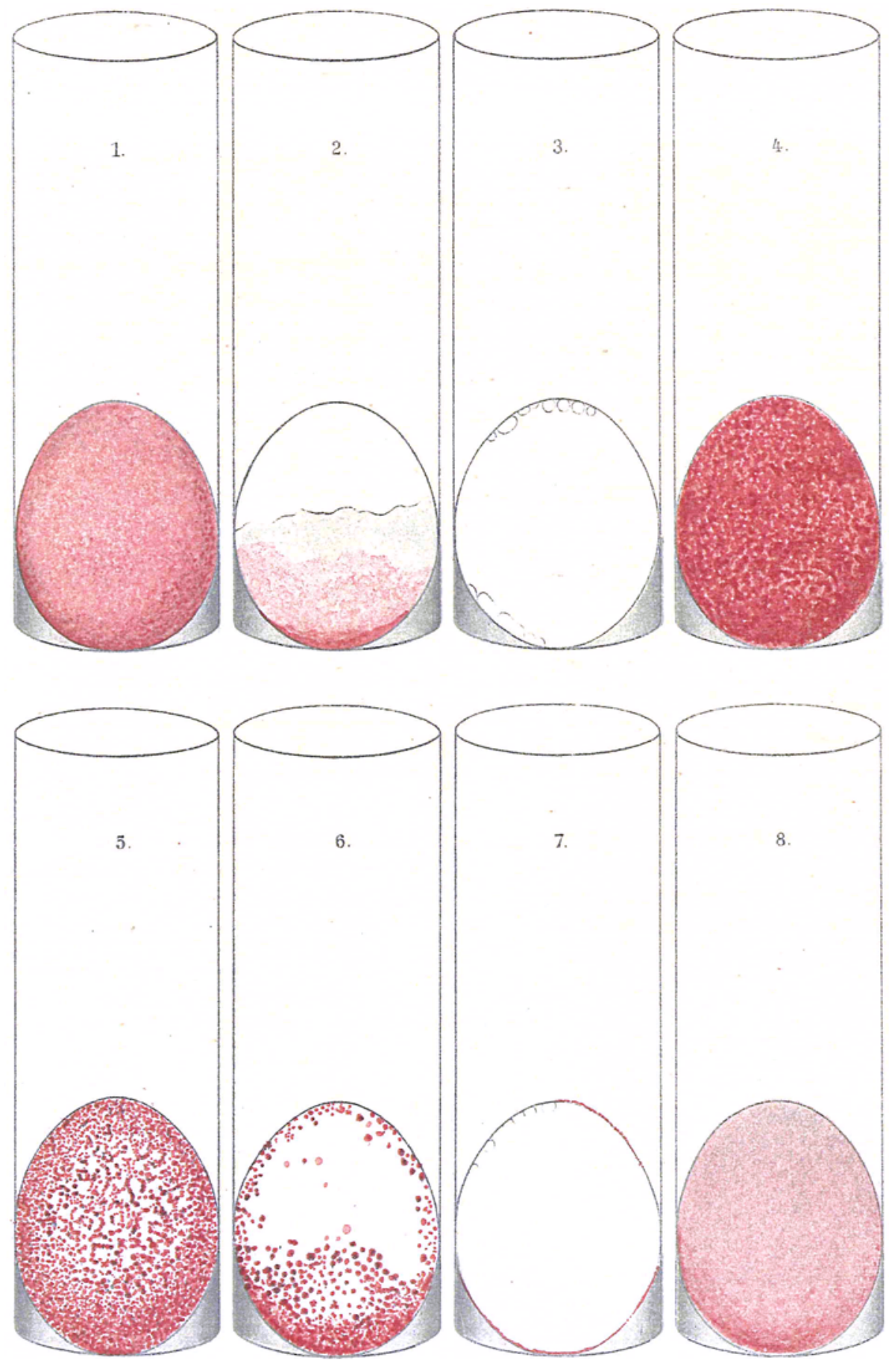

Verlag Veit \& Comp. Levrig
Tatel IV.

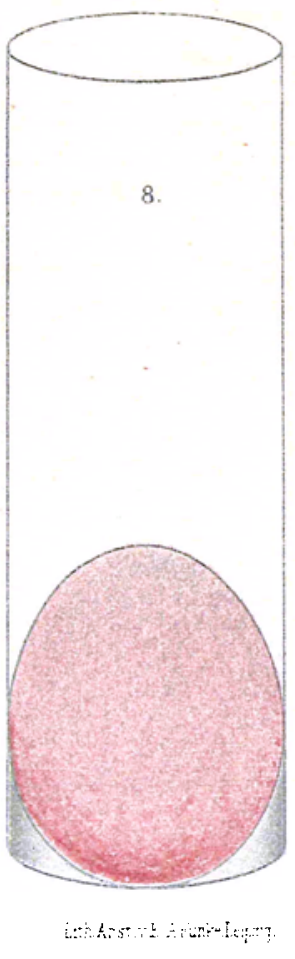

